\title{
Variability of CGRO/EGRET Gamma Ray Sources
}

\author{
M. A. McLaughlin \\ Astronomy Department, Cornell University
}

J. R. Mattox

Astronomy Department, University of Maryland; Space Astronomy Program, Universities Space Research Association

J. M. Cordes

Astronomy Department, Cornell University

D. J. Thompson

Code 661, Laboratory for High Energy Astrophysics, NASA Goddard Space Flight Center

\section{Introduction}

We have developed a method for quantifying the flux variabilities of high energy gamma ray sources. We have applied this method to 128 sources listed in the second catalog of sources detected with the Energetic Gamma Ray Experiment Telescope (EGRET) (Thompson et. al. 1995). These sources include the Large Magellanic Cloud, 5 pulsars, 41 active galactic nuclei (AGN), and 81 sources not yet identified with known objects.

\section{Analysis}

Our data include photon maps ( $\mathrm{E}>100 \mathrm{MeV}$ ) from 134 EGRET viewing periods spanning a roughly $3 \frac{1}{2}$ year period. Each source was observed in 3-20 viewing periods ranging from a few days to 3 weeks in length. For each observation, a source flux was calculated using the method of maximum likelihood (Mattox et al. 1996) which simultaneously estimates the strength of the Galactic and isotropic diffuse emission, and that of a point source distributed as the EGRET point spread function.

We then calculated a weighted mean flux and $\chi^{2}$ value for each source. To determine variability, we find the probability that a $\chi^{2}$ value greater than the empirical $\chi^{2}$ could occur by chance for an intrinsically nonvariable source. This probability, $Q$, is defined in Press et al. (1992). We define the variability index, $V=-\log Q$. Thus, a larger $V$ corresponds to stronger evidence for variability. We classify sources as nonvariable if $V<0.5$, uncertain if $0.5 \leq V<1$, or variable if $V>1$. 


\section{Results}

Of the 128 sources, 54 are found to be nonvariable, 27 have uncertain variability, and 47 are found to be variable. The 54 sources in the nonvariable group include 3 pulsars, $11 \mathrm{AGN}$, and 40 unidentified sources. The 47 variable sources include the LMC, the Crab pulsar, 23 AGN, and 22 unidentified sources. The 27 sources not included in either variable or nonvariable groups include 1 pulsar, 7 AGN, and 19 unidentified sources.

We find that all Galactic pulsars are consistent with being nonvariable. All high flux, identified $A G N$ and many low flux, identified $A G N$ are variable. We find that the apparent nonvariability of the remaining AGN is at least partly due to decreased sensitivity to variability in low flux sources. We recognize populations of both variable and nonvariable unidentified sources.

We have examined the latitude distributions of the various source populations. All pulsars are located in the plane of the Galaxy, as expected. While we expect an isotropic AGN distribution, we find an excess of AGN at high latitudes. This excess can be adequately explained by accounting for decreased EGRET sensitivity at low latitudes. Both variable and nonvariable unidentified sources are in excess at low latitudes.Many low flux, nonvariable, unidentified sources may result from the uncertainty in the Galactic diffuse model at low latitudes. However, the higher flux, nonvariable, unidentified sources at low latitudes represent a definite source population and are excellent Galactic pulsar candidates.

While an uncertain Galactic diffuse model could produce spurious nonvariable sources, it can not explain the excess of variable, unidentified sources at low latitudes. Because decreased EGRET sensitivity at low latitudes sufficiently explains the distribution of identified AGN, these variable, unidentified sources are unlikely to be AGN. Furthermore, the variability of these sources is inconsistent with the steadiness of the 5 known pulsars. Hence, these low latitude, variable gamma ray objects cannot be easily related to any known source population. This suggests that either pulsars can produce variable gamma rays under special circumstances, or that a new class of Galactic gamma ray sources exists.

A longer version of this paper has been submitted to ApJ.

Acknowledgments. M. A. McLaughlin acknowledges support from an NSF fellowship. J. R. Mattox acknowledges support from NASA Grants NAG5-2833 and NAGW-4761. This research was supported by NASA through a GRO grant to Cornell University.

\section{References}

Mattox, J.R. et al. 1996, ApJ, in press

Press, W. H., Teukolsky, S. A., Vetterling, W. T. \& Flannery, B. P. 1992, Numerical Recipes: The Art of Scientific Computing,(Cambridge: Cambridge University Press)

Thompson, D. J. et al. 1995, ApJS, 101, 259 